\title{
Depolarization of backscattered linearly polarized light
}

\author{
L.F. Rojas-Ochoa ${ }^{(1)}$, D. Lacoste ${ }^{(2)}$, R. Lenke ${ }^{(3)}$, P. Schurtenberger ${ }^{(1)}$ and F. Scheffold $^{*(1)}$ \\ (1) Department of Physics, University of Fribourg, CH-1700 Fribourg, Switzerland, \\ (2) Physico-Chimie Théorique ESPCI, 10 Rue Vauquelin, 75231 Paris Cedex 05, France and \\ (3) Fakultät für Physik, Universität Konstanz, D-78547 Konstanz,Germany \\ and Carl Zeiss Laser Optics GmbH, D-73446 Oberkochen, Germany
}

(Dated: October 24, 2018)

We formulate a quantitative description of backscattered linearly polarized light using an extended photon diffusion formalism taking explicitly into account the scattering anisotropy parameter $g$ of the medium. From diffusing wave spectroscopy measurements the characteristic depolarization length for linearly polarized light $\ell_{p}$ is deduced. We investigate the dependance of this length on the scattering anisotropy parameter $g$ spanning an extended range from -1 (backscattering) to 1 (forward scattering). Good agreement is found with Monte Carlo simulations of multiply scattered light.

(C) 2018 Optical Society of America

OCIS codes: $290.4210,030.5620,260.5430$

\section{Introduction}

Polarized light scattered many times in a random medium leaves the sample partially depolarized. Unfortunately, despite its importance in areas like biomedical optical imaging, coherent backscattering or dynamic spectroscopy 1.2 .3 .4 , the depolarization of light in a random medium is still not completely understood due to the complexity of vector wave multiple scattering (as compared to the much simpler problem of scalar wave propagation). Previous attempts have mainly focussed on isotropic (Rayleigh) scattering ${ }^{5}$, or on the depolarization of circularly polarized light $\frac{12}{2}$. Only few studies have discussed specifically the mechanism of depolarization of linearly polarized light in the case where the anisotropy parameter $g=<\cos \theta>$ is different from 0 and furthermore a detailed comparison with experiment has been lacking6.7.8.9.10.11. An accurate description for arbitrary scattering anisotropy is however crucial to analyze the information contained in backscattered light if progress is to be made in applications like remote sensing, photon correlation spectroscopy or optical imaging of biological tissues $1,13,14$.

In this paper we formulate a quantitative description of backscattered linearly polarized light using an extended photon diffusion formalism taking explicitly into account the scattering anisotropy parameter $g$. The details of our model are adjusted by comparison with Monte Carlo simulations of multiply scattered light. We show how the characteristic length of depolarization of incident linearly polarized light $\ell_{p}$ can be deduced from measurements of intensity fluctuations of light scattered from liquid turbid media via Diffusing Wave Spectroscopy (DWS). We can distinguish the following limiting situations for the trans-

\footnotetext{
${ }^{*}$ Corresponding author. E-mail: Frank.Scheffold@unifr.ch
}

port of light and its polarization: isotropic scattering $g \simeq 0$, forward-peaked scattering $g \simeq 1$ and backwardpeaked scattering $g \simeq-1$. The situation of forwardpeaked scattering $g \simeq 1$ is typical of Mie scattering 15 with large particles and of biological tissues, whereas the situation of $g<0$ has only been made possible experimentally recently by tuning the interaction of the light using mesostructured colloidal liquids $\underline{16}$. Here we show that with our additional correction the simple photon diffusion picture successfully describes the distribution of path lengths and the DWS autocorrelation function in the backscattering geometry.

\section{Path lengths distribution for backscattering}

On length scales much larger than the transport mean free path $l^{*}$, the transport of light in a turbid medium can be described by the diffusion approximation. This approximation is connected to the idea of treating the transport of photons as a random walk, characterized by a distribution of path lengths 5.17.18.19. An exact solution of the diffusion equation applied to light transport can be obtained using the method of images. This method takes into account the boundary conditions through two lengths which are both of the order of a transport mean free path: the extrapolation length $z_{e}$ where the flux of the flux of photons vanishes outside the sample and $z_{p}$ which is the location of the photon source (for a more detailed interpretation of $z_{e}, z_{p}$ based on a random walk model see reference (4). The method leads to

$$
P(s)=\frac{\sqrt{3}}{4 \sqrt{\pi \ell^{*}} s^{3 / 2}}\left[z_{p} e^{-\frac{3}{4} \frac{z_{p}^{2}}{\ell^{*} s}}+\left(z_{p}+2 z_{e}\right) e^{-\frac{3}{4} \frac{\left(z_{p}+2 z_{e}\right)^{2}}{\ell^{*} s}}\right] .
$$

which obeys the normalization condition $\int_{0}^{\infty} P(s) d s=1$. Note that the path length is simply related to the number of scattering events $n$ by $s / \ell=n-1$, so that the path length is 0 for single scattering. Here $l$ is the scattering 

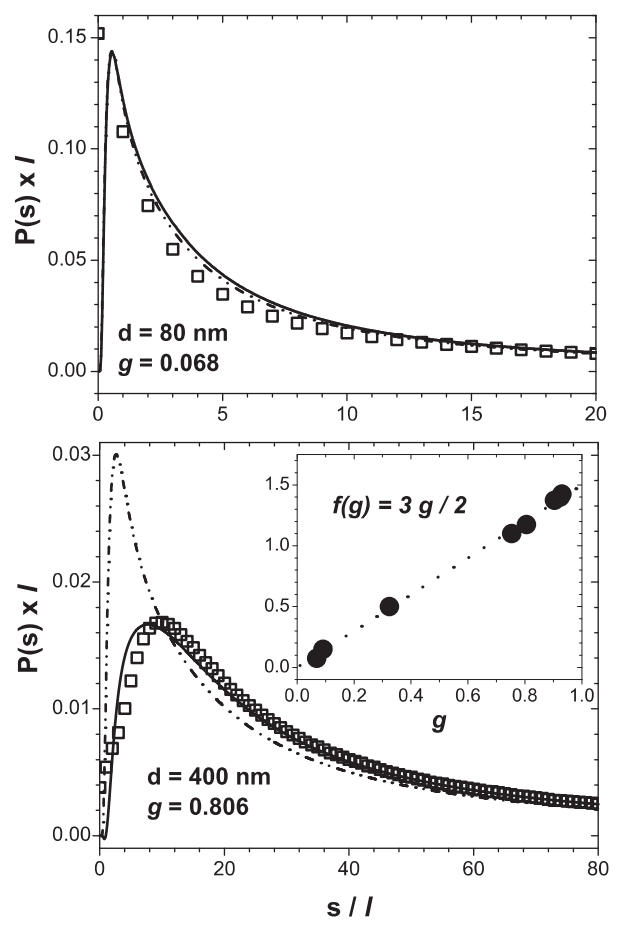

Fig. 1. Normalized path length distribution $P(s) \times l$ for backscattered light from a semi-infinite medium. Symbols: Monte Carlo simulations. Lines: calculations based on Eq. 2 with $f(g)=3 g / 2$ (solid) and $f(g) \equiv 0$ (dashed). Inset: $f(g)$ obtained from Eq. 1 adjusted to fit the simulation results. Wavelength $\lambda_{0}=532 \mathrm{~nm}$, refractive index of the particle $n_{p}=1.59$ and of the solvent $n_{s}=1.332$. Non-reflecting boundary conditions were used.

mean free path. Both quantities, $l$ and $l^{*}$, are related by $l^{*} / l=1 /(1-g)$. The scattering anisotropy parameter $g$ is defined as the average of the cosine of the scattering angle $g=\langle\cos \Theta\rangle$.

To check the validity of Eq. 1] we have performed Monte-Carlo simulations of linearly polarized light reflected from a semi-infinite turbid medium (details about the simulation method can be found in refs. 44 and $(20)$. These simulations use the Mie scattering cross section in the range $0 \leq g \leq 1$ and are able to evaluate numerically an exact path length distribution as a function of the number of scattering events $n$ and polarization. The simulations were done for uncorrelated spherical scatterers (structure function $S(q) \equiv 1$ ) and for a non-reflecting interface characterized by $z_{p} \approx \ell^{*}$ and $z_{e} \approx 0.7 \ell^{*}$. Values of $\lambda_{0} / n_{s}=532 \mathrm{~nm}$ for the incident wavelength, $n_{p}=1.59$ for the refractive index of the particle and $n_{s}=1.332$ for solvent refractive index were used. In Fig. [1 we compare the results of the simulations with the prediction of the method of images according to Eq. 1] We see clearly in this figure that the method of images provides an excellent description of the path length distribution for the case of isotropic scattering $(g \equiv 0)$, but that the method fails to give an equally good description for the case of anisotropic scattering corresponding to $g \simeq 0.806$.

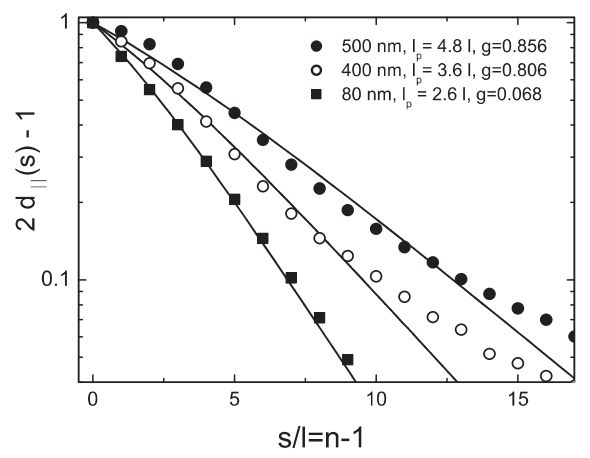

Fig. 2. Depolarization of multiply scattered light. $2 d_{\|}(s)-1$ from theory (Eq.(4) solid lines) and simulation (symbols). Excellent agreement if found for Rayleigh scatterers while for larger particles the agreement becomes somewhat less good.

The disagreement in the latter case is not surprising as the diffusion approximation is known to overestimate the contribution from the short paths of the distribution, the error becoming more and more severe as the anisotropy of scattering increases. One way to improve the distribution of paths length of Eq. 1 is by introducing a cutoff in the distribution as suggested by Mackintosh and $\mathrm{John}^{7}$. Here we extend their approach by taking into account explicitly the scattering anisotropy factor $g$ (with $\left.\int_{0}^{\infty} P_{\text {corr }}(s) d s=1\right)$ :

$$
P_{\text {corr }}(s) \propto P(s) \cdot\left[1-f(g) e^{-s / \ell^{*}}\right]
$$

According to Mackintosh and John ${ }^{7} f(1)$ is of order unity and for isotropic scattering $(g \rightarrow 0)$ there is no correction $f(0)=0$. Using the corrected distribution of path lengths to fit the simulation results, we have found that the function $f$ is well approached by a linear dependance $f(g)=3 g / 2$ which we assume to be valid also for $g<0$. As can be seen in figure 1he use of the correction factor significantly improves the prediction of Eq. 1 We note that, alternatively, for the case of forward-peaked scattering $g \simeq 1$, other schemes of approximations have been suggested. For example the recent Ref ${ }^{11}$ reports that the Fokker-Plank equation provides a better description than does the (uncorrected) diffusion approximation for forward-peaked scattering.

\section{Depolarization length for linear polarization}

Incident polarized light looses its polarization in random multiple scattering 5.6 .7 .8 .10 . For linearly polarized light, only two configurations ( $\|$ and $\perp$ ) need to be considered (for isotropic samples). Physically, in the $\|$ geometry more photons are detected for short paths as compared to the unpolarized case. In a seminal paper Akkermanns et al.$^{\frac{5}{6}}$ found that the path length distribution for the two 
configurations can be written as

$$
P_{\|, \perp}(s)=d_{\|, \perp}(s) \cdot P(s)
$$

with the depolarization ratio given by

$$
\begin{aligned}
& d_{\|}(s)=\frac{1+2 e^{-s / \ell_{p}}}{2+e^{-s / \ell_{p}},} \\
& d_{\perp}(s)=\frac{1-e^{-s / \ell_{p}}}{2+e^{-s / \ell_{p}}},
\end{aligned}
$$

in terms of the characteristic length of depolarization for linearly polarized light $\ell_{p}$. For point like scatterers $(g=$ 0 ) Akkermans et al. obtained $\ell_{p}=\ell / \ln (10 / 7) \cong 2.804 \ell^{\underline{5}}$. We find good agreement between Eq 3 and our numerical simulations with $\ell_{p}$ as an adjustable parameter (Fig.??). For large particles (and therefore large $g$ ) the agreement is somewhat less good. However polarization effects in DWS usually are found weak for $g \approx 1$ and therefore we did not attempt to improve the accuracy of Eqs. (44) and (5) (It is worthwhile to note that close to the sample surface very interesting polarization effects, such as butterfly pattern, persist ${ }^{21,22}$ ).

In the limit $s / \ell \gg 1 \mathrm{Eq} .4$ and 5 reduce to :

$$
P_{\|, \perp}(s) \cong\left[\frac{1}{2} \pm \frac{3}{4} e^{-s / \ell_{p}}\right] \cdot P(s)
$$

We consider this expression the most simple generalization since it captures well intermediate path lengths $s / \ell>3$, where polarization effects are important, but at the same time the number of scattering events is already sufficiently large to apply the diffusion approximation.

\section{Polarization dependence of DWS autocorre- lation function}

In transmission geometry the path length distribution can be measured experimentally using pulsed laser beams ${ }^{23}$. In reflection however paths are short and therefore the time resolution is usually not sufficient for such measurements. An alternative way to probe diffuse light propagation is the analysis of temporal fluctuations of the scattered light via photon correlation spectroscopy. This approach, called diffusing wave spectroscopy (DWS), is a sensitive probe to the path length distribution, in particular in reflection geometry ${ }^{2.3}$. The temporal intensity correlation function $g_{2}(t)$ which is measured, is related to the field autocorrelation function by the Siegert relation $g_{1}(t)=\sqrt{1-g_{2}(t)}$. The latter is directly related to the path length distribution:

$$
g_{1}(t)=\int_{0}^{\infty} P(s) e^{-2\left(t / \tau_{0}\right) s / \ell^{*}} d s .
$$

In our case the characteristic relaxation time for diffusive particle motion (diffusion constant $D$ for free Brownian motion) $\tau_{0}=\left(k_{0}^{2} D\right)^{-1}$ is a known quantity. The path length distribution $\mathrm{P}(\mathrm{s})$, as given by Eq. (1), has been derived for the case of a semi-infinite non-absorbing medium. For real systems, both absorption and limited container size lead to a loss of photons along a given path. For such cases the distribution of path length becomes $P^{\prime}(s)=\exp \left(-s / \ell_{a}\right) P(s)$, where $\ell_{a}$ is the characteristic absorption length of the medium. In this framework, absorption can be taken into account by the modification

$$
6 t / \tau_{0} \rightarrow 6 t / \tau_{0}+3 \ell^{*} / \ell_{a}
$$

in Eq. T. All our experiments correspond to a case where $\ell_{a}$ is much larger than $\ell^{*}$.

The solution for the scalar (polarization independent) path length distribution is well known $\underline{\underline{1}}$ (neglecting absorption): $g_{1}(t)=\left[e^{-\gamma_{p} x(t)}+e^{-\left(\gamma_{p}+2 \gamma_{e}\right) x(t)}\right] / 2$, where $x(t)=\sqrt{6 t / \tau_{0}}, \gamma_{p}=z_{p} / \ell^{*}$ and $\gamma_{e}=z_{e} / \ell^{*}$. In the limit $x \ll 1$ it reduces to

$$
g_{1}(t)=e^{-\gamma x}
$$

with $\gamma=-\frac{\partial \ln g_{1}}{\partial x}(x=0)=\gamma_{p}+\gamma_{e}$. Note that this expression is independent of $\ell^{*}$ and that for the nonreflecting interface that we have considered $\gamma=1+$ $z_{e} / \ell^{*}=5 / 3$. All different paths of length $s$ contribute to $g_{1}(t)$. Short paths predominantly contribute to the long time decay and long paths contribute to the short time decay. Clearly polarization effects modify the path length distribution and therefore strongly influence the decay of $g_{1}(t)$. Interestingly the shape of $g_{1}(t)$ remains more or less unchanged. In most previous studies Eq. 9 though derived for the scalar case, has been applied also with polarized light. Although $\gamma$ is in principle a well defined constant, it has been treated in the literature as an adjustable parameter to explain the polarization dependence of the correlation function. Values of $\gamma_{\perp, \|}$ in a range 1 to 3 have been reported depending on detected polarization state, particle size and concentration.1.6.24.

Rather than to adjust the parameter $\gamma$ we take into account the polarization via Eq. 6 and find:

$$
\begin{aligned}
g_{1 \perp}(t) & =\frac{\int P_{\perp}(s) e^{-2\left(t / \tau_{0}\right) s / \ell^{*} d s}}{P_{\perp}(s) d s} \\
g_{1 \|}(t) & =\frac{\int P_{\|}(s) e^{-2\left(t / \tau_{0}\right) s / \ell^{*} d s}}{P_{\|}(s) d s} .
\end{aligned}
$$

Introducing the function $h(x)=\left(e^{\left(-\gamma_{p} x\right)}+\right.$ $\left.e^{-\left(\gamma_{p}+2 \gamma_{e}\right) x}\right) / 2$, the correlation functions take the form (neglecting absorption)

$g_{1, \perp}(t)=\frac{h\left[x_{1}(t)\right]-\frac{3}{2} h\left[y_{1}(t)\right]-\frac{3 g}{2} h\left[x_{2}(t)\right]+\frac{9 g}{4} h\left[y_{2}(t)\right]}{h\left[x_{1}(0)\right]-\frac{3}{2} h\left[y_{1}(0)\right]-\frac{3 g}{2} h\left[x_{2}(0)\right]+\frac{9 g}{4} h\left[y_{2}(0)\right]}$,

$g_{1, \|}(t)=\frac{h\left[x_{1}(t)\right]+\frac{3}{2} h\left[y_{1}(t)\right]-\frac{3 g}{2} h\left[x_{2}(t)\right]-\frac{9 g}{4} h\left[y_{2}(t)\right]}{h\left[x_{1}(0)\right]+\frac{3}{2} h\left[y_{1}(0)\right]-\frac{3 g}{2} h\left[x_{2}(0)\right]-\frac{9 g}{4} h\left[y_{2}(0)\right]}$, 


\begin{tabular}{|r|c|c|}
\hline $\mathrm{d}(\mathrm{nm})$ & $\Phi(\%)$ & $g$ \\
\hline $80 \pm 5$ & 3.9 & 0.04 \\
$92 \pm 15$ & 4.1 & 0.05 \\
$168 \pm 5$ & $2.0 \rightarrow 30.0$ & $0.29 \rightarrow-0.13$ \\
$350 \pm 12$ & 1.9 & 0.74 \\
$400 \pm 8$ & 1.9 & 0.80 \\
$720 \pm 14$ & 1.9 & 0.90 \\
$1000 \pm 51$ & 2.0 & 0.92 \\
$1500 \pm 53$ & 2.0 & 0.92 \\
$114 \pm 10$ & $2.5 \rightarrow 7.4$ & $-0.25 \rightarrow-0.78$ \\
\hline
\end{tabular}

Table 1. Polystyrene spheres used in the experiments. Diameter $d$ as obtained from dynamic light scattering, volume fraction $\Phi$ and scattering anisotropy parameter $g$. The latter was obtained either from Mie calculations (for hard sphere interactions as described in 16.24) or direct measurements (for the $d=114 \mathrm{~nm}$ charged spheres as described in 16 ) .

where $x_{1}(t)=\sqrt{6 t / \tau_{0}}, x_{2}(t)=\sqrt{6 t / \tau_{0}+3}, y_{1}(t)=$ $\sqrt{6 t / \tau_{0}+3 \ell^{*} / \ell_{p}}$ and $y_{2}(t)=\sqrt{6 t / \tau_{0}+3+3 \ell^{*} / \ell_{p}}$. This set of equations provide a direct relation between measurements detecting $\perp$ or $\|$ polarized light thereby eliminating the need to introduce two adjustable parameters $\gamma_{\perp, \|}$. We carried out a series of dynamic multiple scattering experiments to follow the polarization memory of the reflected light intensity. Experiments were realized as described in $^{24}$ but with $\lambda=532 \mathrm{~nm}$. The sample cells were suspended in a water bath to suppress reflections and to maintain a constant temperature of $T=22^{\circ} \mathrm{C}$. All samples used were made from monodisperse polystyrene particles $(\mathrm{n}=1.595)$ suspended in water $(\mathrm{n}=1.332)$, except for one case $(d=114 \mathrm{~nm})$ where we used a mixture of water and ethanol16 $(\mathrm{n}=1.365)$. A detailed description of all samples is given in table 1

In Fig. 3 we show a comparison between experiments and our theoretical expressions. For clarity the data is normalized and plotted as a function of $x=$ $\sqrt{6 t / t_{0}+3 l^{*} / l_{a}}-\sqrt{3 l^{*} / l_{a}}$ thus removing contributions from absorption at $x<0.1$ (Eq. (8)) (The absorption length $l_{a}$ has been chosen such that $\ln _{1}(x)$ scales linearly at small $x$-values). We find that the theory describes our data very well, with the polarization length $\ell_{p}$ being the only adjustable parameter. In particular the availability of suspensions with negative $g$ values allows a rigorous test of the model over the whole interval $-1 \leq g \leq 1$. Note that we use the theoretically predicted values $\gamma_{p}, \gamma_{e}$ and do not adjust them to fit the data (as done in all previous work). Depolarization lengths obtained from fits (Eq. 11]) to the DWS data and from numerical simulations (Fig.?? ) are presented in Fig. 4 We note again that both DWS experiments $\left(g_{1}, g_{1 \|}\right)$ are well characterized by a single $\ell_{p}$ even for the most extreme case of

$$
g \rightarrow-1
$$

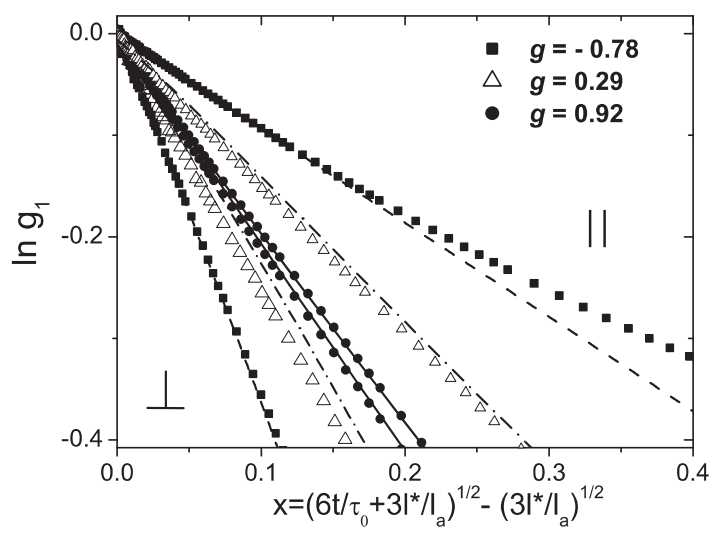

Fig. 3. DWS autocorrelation function for different $g$ values detecting polarized and depolarized light. Lines: calculations based on Eq. 11] with $\ell_{p}$ adjusted to best fit the data. $\left(g=-0.78: \ell^{*} / \ell_{a}=0.004, \ell_{p} / \ell^{*}=5.4\right),(g=$ $\left.0.32: \ell^{*} / \ell_{a}=0.0064, \ell_{p} / \ell^{*}=2.21\right),\left(g=0.924, \ell^{*} / \ell_{a}=\right.$ $\left.0.016, \ell_{p} / \ell^{*}=0.713\right)$.

In the limit $g \simeq 0$ we find good agreement with the predicted theoretical value of Akkermans $\ell_{p} / \ell \cong 2.804^{5}$. As the anisotropy parameter $g$ is increased $\ell_{p} / \ell$ slowly increases as well. In the case of forward-peaked scattering $g \simeq 1$, we find $\ell_{p} \simeq \ell^{*}$ (see also refs. (으) and (9) ). This means that as $g$ approaches 1 an increasing number of scattering events is necessary to depolarize backscattered light. Since $\ell_{p} / \ell^{*}$ remains constant for $g \rightarrow 1$ the ratio $\ell_{p} / \ell$ has to increase sharply, as shown in Figure $4 \mathrm{~b}$. In the case of backward-peaked scattering $g \simeq-1$ however, the number of scattering events needed to depolarize remains virtually unchanged. The characteristic length scale of depolarization is still the scattering mean free path $\ell$ (and not $\ell^{*}$ !) as in the case of point-like scatterers. Therefore it is not surprising to see that in Fig. 团 $\ell_{p}$ scales as in the Rayleigh limit i.e $\ell_{p} \simeq 3 \ell$ even for a transport mean free path $\ell^{*} \simeq \ell / 2$. When $\ell_{p} / \ell^{*}$ is plotted as a function of $g$ we find an almost linear behavior over the full accessible range. At this point our understanding of $\ell_{p}$ is limited to the particular cases $(g=0, g=1$, and $g=-1)$, a more detailed microscopic approach would be required to explain the complete dependance of $\ell_{p}$ on $g$, or the dependance on other single particle optical properties.

\section{Depolarization ratio of backscattered intensi- ties}

An alternative way to study polarization of multiple backscattered waves is through the (full intensity) depolarization ratio:

$$
d=\frac{I_{\|}-I_{\perp}}{I_{\|}+I_{\perp}}
$$




$$
d=\frac{\int\left[P_{\|}(s)-P_{\perp}(s)\right] d s}{\int\left[P_{\|}(s)+P_{\perp}(s)\right] d s} \cong \int_{0}^{\infty} \frac{3}{2} e^{-s / \ell_{p}} P(s) d s,
$$

from where we identify a $s$-dependent depolarization ratio $d(s)$ :

$$
d(s) \cong \frac{3}{2} e^{-s / \ell_{p}} .
$$

Since $P_{\|}(s)+P_{\perp}(s)=P(s)$ and $\int_{0}^{\infty} P(s) d s=1$, therefore:

$$
\begin{aligned}
d & =\int_{0}^{\infty} P(s) d(s) d s \\
& \cong \frac{3}{4}\left(e^{-\gamma_{p} \sqrt{3 \ell^{*} / \ell_{p}}}+e^{-\left(\gamma_{p}+2 \gamma_{e}\right) \sqrt{3 \ell^{*} / \ell_{p}}}\right) .
\end{aligned}
$$

In Figure 4r we show the experimental values of $d$. Again the agreement is excellent over the full accessible range. The data comes very close to the predicted values in the three particular cases: $d=0.33$ for Rayleigh scattering $(g=0), d=0.14$ for forward-peaked scattering $(g=1)$ and $d=0.49$ for backward peaked scattering $(g=-1)$.

\section{Conclusion}

In this paper, we have shown how to describe the effect of the scattering anisotropy on the depolarization of linearly polarized light, and how to use Diffusing Wave Spectroscopy (DWS) to determine the characteristic depolarization properties. By means of numerical simulations, we checked the limit of validity of the diffusion approximation when the scattering anisotropy $g$ is increased, and we have shown how to correct the predictions by means of an anisotropy dependent cutoff for the path length distribution $P(s)$. We discuss for the first the time the dependence of the characteristic depolarization length over the full range of possible values of $g$ including the unusual case of negative $g$ values. In our description the extrapolation length $\gamma$ is a well defined constant as required by diffusion theory. Our work thus clarifies the meaning of $\gamma$, subject to intense discussion in the past ${ }^{25.26}$. Since our description only uses a single adjustable parameter, it is now possible to fully characterize backscattered light with polarization resolved measurements. We think that this approach can strongly benefit applications in the field of soft and bio material analysis, as well as diffuse light imaging techniques $1.11,27$.

\section{Acknowledgments}

We thank D. Pine, M. Cloitre and A. Maggs and F. Jaillon for discussions. Financial support from the Swiss National Science foundation is gratefully acknowledged.

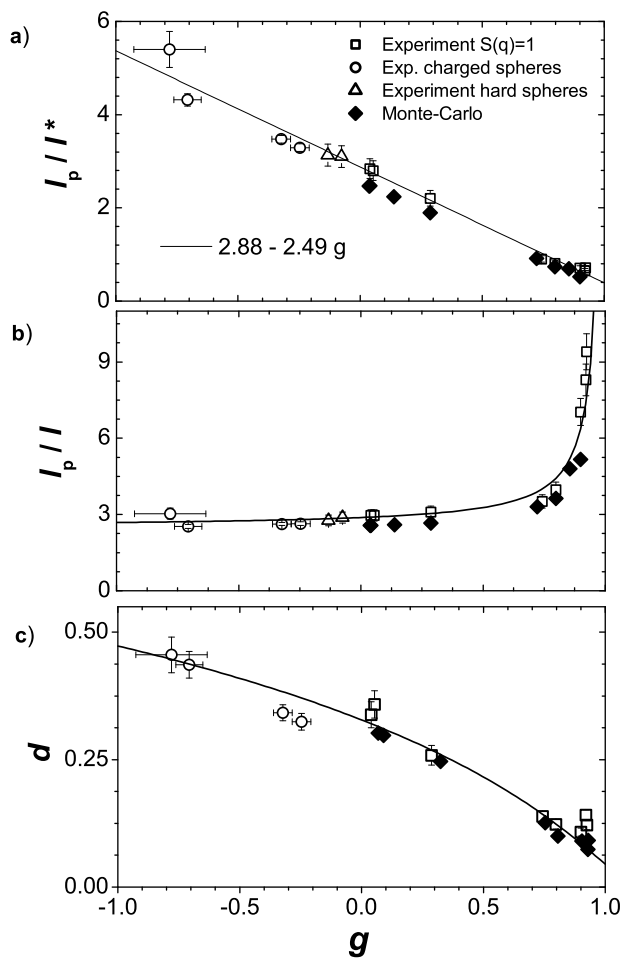

Fig. 4. a) and b) Depolarization length $\ell_{p}$ from DWS measurements and Monte-Carlo simulations. Squares: measurements for different particle sizes (random particle configuration $S(q) \equiv 1$ ). Circles: Strongly interacting charged particles ${ }^{16}$. Triangles: Hard sphere data from reference 24. Diamonds: Monte-Carlo simulations. c) Depolarization ratio directly obtained from the measured intensities. Solid lines: calculated from the linear fit to $\ell_{p} / \ell^{*}$ shown in panel a $)^{4.20}$.

\section{References}

1. D. A. Weitz and D. J. Pine, in Dynamic Light Scattering, ed. by W. Brown, Oxford U. Press; New York(1993), Chap. 16, pp. 652-720.

2. G. Maret and P.E. Wolf, "Multiple Light-Scattering From Disordered Media - The Effect Of BrownianMotion Of Scatterers", Z. Phys. B 65, 409-431 (1987).

3. D. J. Pine, D. A. Weitz, P. M. Chaikin, and E. Herbolzheimer, "Diffusing-Wave Spectroscopy", Phys. Rev. Lett. 60, 1134-1137 (1988).

4. R. Lenke and G. Maret, in Multiple Scattering of Light: Coherent Backscattering and Transmission, ed. by W. Brown, Gordon and Breach Science Publishers; Reading U. K. (2000), pp. 1-72.

5. E. Akkermans, P. E. Wolf, R. Maynard and G. Maret, "Theoretical-Study Of The Coherent Backscattering Of Light By Disordered Media", J. Phys. France 49, 77-98 (1988) 
6. F. C. MacKintosh, J. X. Zhu, D. J. Pine and D. A. Weitz, "Polarization Memory Of Multiply Scattered-Light", Phys. Rev. B 40, 9342-9345 (1989).

7. F. C. MacKintosh and S. John, "Diffusing-Wave Spectroscopy And Multiple-Scattering Of Light In Correlated Random-Media", Phys. Rev. B 40, 23832406 (1989).

8. D. Bicout, C. Brosseau, A. S. Martinez, and J. M. Schmitt, "Depolarization of multiply scattered waves by spherical diffusers: Influence of the size parameter", Phys. Rev. E, 49, 1767-1770, (1994).

9. D. Lacoste, V. Rossetto, F. Jaillon and H. SaintJalmes, submitted

10. D. A. Zimnyakov, Y. P. Sinichkin, P. V. Zakharov and D. N. Agafonov, "Residual polarization of noncoherently backscattered linearly polarized light: the influence of the anisotropy parameter of the scattering medium", Waves Random Media 11, 395412 (2001).

11. A. D. Kim and J. B. Keller, "Light propagation in biological tissue", J. Opt. Soc. Am. A, 20 (1), 92-98, (2003).

12. E. E. Gorodnichev, A. I. Kuzovlev and D. B. Rogozkin, "Diffusion of circularly polarized light in a disordered medium with large- scale inhomogeneities", JETP Letters, 68(1), 22-28, (1998).

13. M. Moscoso, J. B. Keller and G. Papanicolaou, "Depolarization and blurring of optical images by biological tissue", J. Opt. Soc. Am. A, 18 (4), 948-960, (2001).

14. P. Sebbah, Waves and imaging through complex media (Kluwer Academic Publishers, Dordrecht ; Boston, 2001).

15. H. C. van de Hulst, Light Scattering by Small Particles, Dover, New York (1981). C. F. Bohren and D. R. Huffman, Absorption and Scattering of Light by Small Particles, Wiley, New York (1983).

16. L.F. Rojas-Ochoa, J.M. Mendez-Alcaraz, J.J. Saenz, P. Schurtenberger and F. Scheffold, "Photonic properties of strongly correlated colloidal liquids", submitted; We note that in present work we could not access concentrations above $\Phi=7.4 \%$ due to the apparent non-egodicity of the scattering signal being much more pronounced in backscattering as compared to the transmission geometry studied before.

17. A. Ishimaru, Wave propagation and scattering in random media, Academic Press, New York (1978).

18. A. Lagendijk and R. Vreeker and P. DeVries, "Influence Of Internal-Reflection On Diffusive Transport In Strongly Scattering Media", Phys. Lett. A 136, 81-88 (1989).

19. J. X. Zhu and D. J. Pine and D. Weitz, "InternalReflection Of Diffusive Light In Random-Media", Phys. Rev. A 44, 3948-3959 (1991).

20. R. Lenke and G. Maret, "Magnetic field effects on coherent backscattering of light", Eur. Phys. J. B
17, 171-185 (2000).

21. A. C. Maggs and V. Rossetto, "Writhing Photons and Berry Phases in Polarized Multiple Scattering", Phys. Rev. Lett. 87, 253901 (2001).

22. A.H. Hielscher et. al., Optics exp. 1, 441-453 (1997).

23. A.G. Yodh, P.D. Kaplan, D.J. Pine, "Pulsed Diffusing-Wave Spectroscopy - High-Resolution Through Nonlinear Optical Gating", Phys. Rev. B 42, 4744-4447 (1990).

24. L. F. Rojas-Ochoa and S. Romer and F. Scheffold and P. Schurtenberger, "Diffusing wave spectroscopy and small-angle neutron scattering from concentrated colloidal suspensions", Phys. Rev. E 65, 051403 (2002).

25. M. Rosenbluh and M. Hoshen and I. Freund and M. Kaveh, "Time evolution of universal optical fluctuations", Phys. Rev. Lett 58, 2754-2757 (1987).

26. I. Freund and M. Kaveh, "Comment on 'Polarization memory of multiply scattered light"', Phys. Rev. B 45, 8162-8163 (1992); F. C. MacKintosh and J. X. Zhu and D. J. Pine and D. A. Weitz, "Reply to comment on 'Polarization Memory Of Multiply Scattered-Light"', Phys. Rev B 45, 8165-8165 (1992).

27. F. Scheffold, "Particle sizing with diffusing wave, spectroscopy", J. Disp. Sci. Tech 23, 591-599 (2002). 